\title{
ANALYTIC FUNCTIONS AND INTEGRABLE HIERARCHIES-CHARACTERIZATION OF TAU FUNCTIONS
}

\author{
LEE-PENG TEO
}

\begin{abstract}
We prove the dispersionless Hirota equations for the dispersionless Toda, dispersionless coupled modified KP and dispersionless KP hierarchies using an idea from classical complex analysis. We also prove that the Hirota equations characterize the tau functions for each of these hierarchies. As a result, we establish the links between the hierarchies.
\end{abstract}

\section{INTRODUCTION}

Dispersionless integrable hierarchies have been under active research in recent years (see, e.g. [14, 15, 21, 20, 22, 23]). One of the reasons is due to its close relation with the other area of mathematics and physics, such as topological field theory, string theory, 2D-gravity, matrix models and conformal maps (see, e.g. [18, 19, 8, 11, 1, 2, 3, 25, 13, 4, 26, 16]). The tau functions of the dispersionless integrable hierarchies play an important role in topological field theories ( 9, 10, for they give solutions to the so-called WDVV equation. Conversely, in 4, it was proved that the tau functions of the dispersionless KP (dKP) and dispersionless Toda (dToda) hierarchies satisfy the associativity equation. One of the main ingredients of the proof of [4] is the dispersionless Hirota equations satisfied by the dKP and dToda hierarchies. The dispersionless Hirota equation for the dKP hierarchy was first derived by Takasaki and Takebe [23] as the dispersionless limit of the differential Fay identity. Later, the Hirota equation was further studied by Carroll and Kodama [5]. In connection to conformal mappings which give rise to solutions of the dToda hierarchy, Wiegmann, Zabrodin et al derive the Hirota equations for the dToda hierarchy [25, 16, 13]. In this paper, we point out that these Hirota equations are closely related to some concepts in classical complex analysis, namely Faber polynomials and Grunsky coefficients.

In Section 2, we review some concepts from classical complex analysis. We define some classes of formal power series which appear in dKP, dcmKP

2000 Mathematics Subject Classification. Primary 37K10, 37K20; Secondary 32A05, $30 \mathrm{~B} 10$.

Key words and phrases. Dispersionless integrable hierarchies, tau functions, algebraic analysis, Grunsky coefficients. 
(dispersionless coupled modified KP, see 24]) and dToda hierarchies. We generalize the definition of the Grunsky coefficients and Faber polynomials to these classes of formal power series and review their properties. In Section 3 , we review the dToda, dcmKP and dKP hierarchies and their tau functions. We derive the dispersionless Hirota equations by establishing the relation between the tau functions and the Grunsky coefficients. We also prove that the dispersionless Hirota equations for each of these hierarchies uniquely characterize the tau functions of their solutions. As a corollary, we show that some solutions of the dToda hierarchy will give rise to solutions of dcmKP hierarchy, which in turn will give rise to solutions of dKP hierarchy.

\section{Algebraic analysis}

2.1. Spaces of formal power series. We consider the following classes of formal power series :

$$
\begin{aligned}
\tilde{\Sigma} & =\left\{g(z)=b z+b_{0}+\frac{b_{1}}{z}+\ldots=b z+\sum_{n=0}^{\infty} b_{n} z^{-n} ; b \neq 0\right\}, \\
\Sigma & =\left\{g(z)=z+b_{0}+\frac{b_{1}}{z}+\ldots=z+\sum_{n=0}^{\infty} b_{n} z^{-n}\right\}, \\
\Sigma_{0} & =\left\{g(z)=z+\frac{b_{1}}{z}+\frac{b_{2}}{z^{2}}+\ldots=z+\sum_{n=1}^{\infty} b_{n} z^{-n}\right\} .
\end{aligned}
$$

$\tilde{\Sigma}$ can be considered as completion ${ }^{1}$ of the space of analytic functions that fix the point $\infty$ and univalent in a small neighbourhood of $\infty . \Sigma$ and $\Sigma_{0}$ are subspaces of $\tilde{\Sigma}$ consist of formal power series satisfying certain normalization conditions. By post-composing $\tilde{g} \in \tilde{\Sigma}$ with the linear map $z \mapsto(1 / \tilde{b}) z$, we get a function $g$ in $\Sigma$. Further post-composition with the linear map $z \mapsto z-b_{0}$, we get a function $g_{0}$ in $\Sigma_{0}$. As their counterpart, we consider another three classes of formal power series:

$$
\begin{aligned}
\tilde{S} & =\left\{f(z)=a_{1} z+a_{2} z^{2}+a_{3} z^{3}+\ldots=\sum_{n=1}^{\infty} a_{n} z^{n} ; a_{1} \neq 0\right\}, \\
S & =\left\{f(z)=z+a_{2} z^{2}+a_{3} z^{3}+\ldots=z+\sum_{n=2}^{\infty} a_{n} z^{n}\right\}, \\
S_{0} & =\left\{f(z)=z+a_{3} z^{3}+a_{4} z^{4}+\ldots=z+\sum_{n=3}^{\infty} a_{n} z^{n}\right\} .
\end{aligned}
$$

\footnotetext{
${ }^{1}$ The completion is with respect to the filtration $\tilde{\Sigma}_{N}=$ $\left\{g(z)=b z+\sum_{n=N}^{\infty} b_{n} z^{-n}, b \neq 0\right\}$ on $\tilde{\Sigma}$.
} 
$\tilde{S}$ can be considered as completion of the space of analytic functions fixing the point 0 and univalent in a neighbourhood of $0 . S$ and $S_{0}$ are subspaces of $\tilde{S}$ consist of formal power series subjecting to additional normalization conditions. By post-composing $\tilde{f} \in \tilde{S}$ with the linear map $z \mapsto\left(1 / \tilde{a}_{1}\right) z$, we get a function $f \in S$. Further post-composition with the Möbius transformation $z \mapsto z /\left(1+a_{2} z\right)$, we get a function $f_{0} \in S_{0}$. Observe that the map

$$
f \mapsto g(z)=\frac{1}{f\left(\frac{1}{z}\right)}
$$

is a bijection between $\tilde{S}$ and $\tilde{\Sigma}, S$ and $\Sigma, S_{0}$ and $\Sigma_{0}$ respectively.

2.2. Faber polynomials and Grunsky coefficients. We review and generalize some concepts from classical complex analysis. For details, see [17, 12 .

One of the important problems in classical complex analysis is the determination of the upper bound satisfied by the coefficients of $a_{n}$ 's in order that $f \in S$ is univalent on the unit disc. A lot of effort has been devoted to the proof of the famous Bieberbach conjecture (1916) : If $f \in S$ is univalent on the unit disc, then $\left|a_{n}\right| \leq n^{2}$. In the early attempts of the proof, one of the important tools is the Grunsky's inequality and its generalization. In 1939, Grunsky found a sequence of inequalities that should be satisfied by the so called Grunsky coefficients in order that $g \in \Sigma$ is univalent on $\{z|| z \mid>1\}$. Surprisingly, we found this Grunsky coefficients appear everywhere in dispersionless limit of integrable hierarchies (especially in association with tau functions) without being realized its connection to complex analysis. This is the purpose of this paper to point out this connection, and thus give simplified proofs of some of the facts related to dispersionless integrable hierarchies.

First, we introduce the Faber polynomials. For $g \in \tilde{\Sigma}$ that is analytic in a neighbourhood of $\infty$ and $w \in \mathbb{C}$, consider the function $\log \frac{g(z)-w}{b z}$. It defines an analytic function for large $|z|$ and vanishes at $\infty$. Hence it has an expansion at $\infty$ which can be written as

$$
\log \frac{g(z)-w}{b z}=-\sum_{n=1}^{\infty} \frac{\Phi_{n}(w)}{n} z^{-n} .
$$

$\Phi_{n}$ is called the $n$-th Faber polynomial of $g$. Differentiate (2.1) with respect to $z$ and define $\Phi_{0}(w) \equiv 1$, we have

$$
\frac{g^{\prime}(z)}{g(z)-w}=\sum_{n=0}^{\infty} \Phi_{n}(w) z^{-n-1} .
$$

\footnotetext{
${ }^{2}$ This conjecture was completely proved by de Branges in 1984 [7].
} 
Putting $g(z)=b z+b_{0}+\sum_{n=1}^{\infty} b_{n} z^{-n}$ into this expression, we have

$$
b-\sum_{n=1}^{\infty} n b_{n} z^{-n-1}=\left(b z+b_{0}-w+\sum_{n=1}^{\infty} b_{n} z^{-n}\right)\left(\frac{1}{z}+\sum_{n=1}^{\infty} \Phi_{n} z^{-n-1}\right) .
$$

Comparing coefficients, we have

$$
\Phi_{1}(w)=\frac{w-b_{0}}{b}
$$

and the recursion formula

$$
\Phi_{n+1}(w)=\frac{w-b_{0}}{b} \Phi_{n}(w)-\frac{1}{b} \sum_{k=1}^{n-1} b_{n-k} \Phi_{k}(w)-(n+1) \frac{b_{n}}{b},
$$

which can be used to solve for $\Phi_{n}$. From this, we see that $\Phi_{n}(w)$ is a polynomial of degree $n$ of the form

$$
\Phi_{n}(w)=\left(\frac{w-b_{0}}{b}\right)^{n}-n \frac{b_{1}}{b}\left(\frac{w-b_{0}}{b}\right)^{n-2}+\ldots
$$

The first few are given by

$$
\begin{aligned}
& \Phi_{2}(w)=\left(\frac{w-b_{0}}{b}\right)^{2}-2 \frac{b_{1}}{b} \\
& \Phi_{3}(w)=\left(\frac{w-b_{0}}{b}\right)^{3}-3 \frac{b_{1}}{b} \frac{w-b_{0}}{b}-3 \frac{b_{2}}{b}, \\
& \Phi_{4}(w)=\left(\frac{w-b_{0}}{b}\right)^{4}-4 \frac{b_{1}}{b}\left(\frac{w-b_{0}}{b}\right)^{2}-4 \frac{b_{2}}{b} \frac{w-b_{0}}{b}+2\left(\frac{b_{1}}{b}\right)^{2}-4 \frac{b_{3}}{b} .
\end{aligned}
$$

Now we introduce the Grunsky coefficients. If $g \in \tilde{\Sigma}$ is univalent in a neighbourhood $U$ of $\infty$, the function $\log \frac{g(z)-g(\zeta)}{z-\zeta}$ is analytic in the neighbourhood $U \times U$ of $(\infty, \infty)$. Its expansion about $(\infty, \infty)$ has the form

$$
\log \frac{g(z)-g(\zeta)}{z-\zeta}=\log b-\sum_{m=1}^{\infty} \sum_{n=1}^{\infty} b_{m n} z^{-m} \zeta^{-n} .
$$

$b_{m n}$ 's are known as Grunsky coefficients of $g$. They are symmetric, i.e. $b_{m n}=b_{n m}$. If we put $w=g(\zeta)$ into (2.1), we have

$$
\log \frac{g(z)-g(\zeta)}{z-\zeta}=\log b-\sum_{n=1}^{\infty} \frac{1}{n}\left(\Phi_{n}(g(\zeta))-\zeta^{n}\right) z^{-n} .
$$

Compare with (2.3), we have

$$
\Phi_{n}(g(\zeta))=\zeta^{n}+n \sum_{m=1}^{\infty} b_{n m} \zeta^{-m} .
$$

In fact, the Faber polynomials can be uniquely characterized as follows: If $P_{n}(w)$ is a degree $n$ polynomial such that $P_{n}(g(\zeta))-\zeta^{n} \rightarrow 0$ as $\zeta \rightarrow \infty$, then $P_{n}=\Phi_{n}$. 
Since $\Phi_{1}(w)=\left(w-b_{0}\right) / b$, the $n=1$ case of (2.4) gives

$$
g(\zeta)=b \zeta+b_{0}+b \sum_{m=1}^{\infty} b_{1 m} \zeta^{-m}
$$

Hence $b_{1 m}=b_{m 1}=b_{m} / b$. In fact, using the recursion formula for $\Phi_{n}$ and (2.4), we can recursively solve for $b_{n m}$ 's. The first fews are given by

$b_{22}=\frac{b_{3}}{b}+\frac{1}{2}\left(\frac{b_{1}}{b}\right)^{2}, \quad b_{23}=\frac{b_{4}}{b}+\frac{b_{1} b_{2}}{b^{2}}, \quad b_{33}=\frac{b_{5}}{b}+\frac{b_{1} b_{3}}{b^{2}}+\left(\frac{b_{2}}{b}\right)^{2}+\frac{1}{3}\left(\frac{b_{1}}{b}\right)^{3}$.

There is another characterization of the Faber polynomials which plays an important role in our discussion later. Let $z=G(w)=w / b+\sum_{n=0}^{\infty} c_{n} w^{-n}$ be the inverse function of $w=g(z)$ in the neighbourhood $U$ where $g$ is univalent. The coefficients $c_{n}$ can be solved recursively. $c_{0}=-b_{0} / b$ and for $n \geq 1, c_{n}$ is a polynomial in $b, b_{0}, b_{1}, \ldots, b_{n}$. Let $C$ be a simple closed curve winding negatively about the point $\infty$, lying entirely in $U$. Then $C^{\prime}=g(C)$ is also a simple closed curve winding negatively about $\infty$. Observe that the line integral of a function $(1 / 2 \pi i) f$ along $C$ (or $C^{\prime}$ ) amounts to taking residue of $f$ with respect to $\infty$. Hence from (2.2), we have

$$
\Phi_{n}(w)=\frac{1}{2 \pi i} \oint_{C} \frac{g^{\prime}(z) z^{n}}{g(z)-w} d z .
$$

Making a change of variable $z=G(\zeta)$ or equivalently $\zeta=g(z)$, we have

$$
\Phi_{n}(w)=\frac{1}{2 \pi i} \oint_{C^{\prime}} \frac{G(\zeta)^{n}}{\zeta-w} d \zeta
$$

Using the expansion about $\zeta=\infty, G(\zeta)^{n}=\sum_{m=-\infty}^{n} c_{n, m} \zeta^{m}$ and $1 /(\zeta-w)=$ $\sum_{k=0}^{\infty} w^{k} \zeta^{-k-1}$, we obtain immediately $\Phi_{n}(w)=\sum_{m=0}^{n} c_{n, m} w^{m}$. Namely, $\Phi_{n}(w)$ is the polynomial part of $G(w)^{n}$, which we denote by $\left(G(w)^{n}\right)_{\geq 0}$, i.e.

$$
\Phi_{n}(w)=\left(G(w)^{n}\right)_{\geq 0} .
$$

In general, if $A=\sum_{n=-\infty}^{\infty} A_{n} w^{n}$ is a formal power series, and $S$ a subset of integers, we define $(A)_{S}=\sum_{n \in S} A_{n} w^{n}$.

The Grunsky coefficients are generalized to a pair of functions $f$ and $g$ as follows. Let $f \in \tilde{S}$ be univalent in a neighbourhood $V$ of 0 and $g \in \Sigma$ be univalent in a neighbourhood $U$ of $\infty$. We say that $(f, g)$ are disjoint relative to $(U, V)$ if the sets $f(V)$ and $g(U)$ are disjoint. In this case, the functions

$$
\log \frac{g(z)-g(\zeta)}{z-\zeta}, \quad \log \frac{g(z)-f(\zeta)}{z}, \quad \log \frac{f(z)-f(\zeta)}{z-\zeta}
$$


are analytic in $U \times U, U \times V$ and $V \times V$ respectively. Hence we can write down their series expansion about $(\infty, \infty),(\infty, 0)$ and $(0,0)$ respectively:

$$
\begin{aligned}
\log \frac{g(z)-g(\zeta)}{z-\zeta} & =-\sum_{m=1}^{\infty} \sum_{n=1}^{\infty} b_{m n} z^{-m} \zeta^{-n}, \\
\log \frac{g(z)-f(\zeta)}{z} & =-\sum_{m=1}^{\infty} \sum_{n=0}^{\infty} b_{m,-n} z^{-m} \zeta^{n}, \\
\log \frac{f(z)-f(\zeta)}{z-\zeta} & =-\sum_{m=0}^{\infty} \sum_{n=0}^{\infty} b_{-m,-n} z^{m} \zeta^{n} .
\end{aligned}
$$

Obviously, when $m, n$ are both positive or both negative, $b_{m n}=b_{n m}$. Hence for $m \geq 0, n>0$, we define $b_{-m, n}=b_{n,-m}$. Letting $\zeta=0$ in (2.7) and (2.8), we obtain

$$
\log \frac{g(z)}{z}=-\sum_{m=1}^{\infty} b_{m, 0} z^{-m}, \quad \log \frac{f(z)}{z}=-\sum_{m=0}^{\infty} b_{-m, 0} z^{m} .
$$

In particular, $b_{00}=-\log a_{1}$. We define the generalized Faber polynomials $\Psi_{n}(w)$ for $f$ by

$$
\log \frac{w-f(z)}{w}=\log \frac{f(z)}{a_{1} z}-\sum_{n=1}^{\infty} \frac{\Psi_{n}(w)}{n} z^{n} .
$$

To see the relations between $\Psi_{n}$ and the Grunsky coefficients, we define the function $g_{f} \in \tilde{\Sigma}$ by

$$
g_{f}(z)=\frac{1}{f\left(\frac{1}{z}\right)}=\frac{z}{a_{1}}-\frac{a_{2}}{a_{1}^{2}}+\ldots
$$

Using the second equation in (2.9), equations (2.7), (2.8) and (2.10) can be rewritten in terms of $g_{f}$ :

$$
\begin{aligned}
\log \left(1-\frac{1}{g(z) g_{f}(\zeta)}\right) & =-\sum_{m=1}^{\infty} \sum_{n=1}^{\infty} b_{m,-n} z^{-m} \zeta^{-n}, \\
\log \frac{g_{f}(z)-g_{f}(\zeta)}{z-\zeta} & =-\log a_{1}-\sum_{m=1}^{\infty} \sum_{n=1}^{\infty} b_{-m,-n} z^{-m} \zeta^{-n}, \\
\log \frac{g_{f}(z)-\frac{1}{w}}{z} & =-\log a_{1}-\sum_{n=1}^{\infty} \frac{\Psi_{n}(w)}{n} z^{-n} .
\end{aligned}
$$

Hence, $b_{-m,-n}$ 's are Grunsky coefficients of $g_{f}$ and $\Psi_{n}(w)$ is a polynomial of degree $n$ in $1 / w$. If we denote by $z=G_{f}(w)$ the inverse of $w=g_{f}(z)$, and by $z=F(w)$ the inverse of $w=f(z)$, then

$$
F(w)=\frac{1}{G_{f}\left(\frac{1}{w}\right)} .
$$


Equation (2.5) implies that

$$
\Psi_{n}\left(w^{-1}\right)=\left(G_{f}(w)^{n}\right)_{\geq 0}
$$

or equivalently

$$
\Psi_{n}(w)=\left(G_{f}\left(w^{-1}\right)^{n}\right)_{\leq 0}=\left(F(w)^{-n}\right)_{\leq 0} .
$$

Now we derive the counterparts of (2.4). First, compare (2.13) to (2.1) and (2.4), we have

$$
\Psi_{n}\left(g_{f}(\zeta)^{-1}\right)=\zeta^{n}+n \sum_{m=1}^{\infty} b_{-n,-m} \zeta^{-m}
$$

or equivalently

$$
\Psi_{n}(f(\zeta))=\zeta^{-n}+n \sum_{m=1}^{\infty} b_{-n,-m} \zeta^{m}
$$

Next, we put $w=f(\zeta)$ into (2.1) and compare with (2.7), we obtain

$$
\Phi_{n}(f(\zeta))=n \sum_{m=0}^{\infty} b_{n,-m} \zeta^{m}
$$

Finally, putting $w=g(\zeta)$ into (2.10), we have

$$
\log \frac{g(\zeta)-f(z)}{\zeta}=\log \frac{g(\zeta)}{\zeta}+\log \frac{f(z)}{a_{1} z}-\sum_{n=1}^{\infty} \frac{\Psi_{n}(g(\zeta))}{n} z^{n} .
$$

Compare with (2.7) and using equations in (2.9), we have

$$
\Psi_{n}(g(\zeta))=-n b_{-n, 0}+n \sum_{m=1}^{\infty} b_{m,-n} \zeta^{-m} .
$$

For the convenience of next section, we gather again the formulas (2.9), and the formulas of the Faber polynomials in terms of the Grunsky coefficients (2.4), (2.15), (2.16), (2.17).

$$
\begin{array}{ll}
\log \frac{g(z)}{z}=-\sum_{m=1}^{\infty} b_{m, 0} z^{-m}, \quad \log \frac{f(z)}{z}=-\sum_{m=0}^{\infty} b_{-m, 0} z^{m} \\
\Phi_{n}(g(\zeta))=\zeta^{n}+n \sum_{m=1}^{\infty} b_{n m} \zeta^{-m}, \quad \Phi_{n}(f(\zeta))=n b_{n, 0}+n \sum_{m=1}^{\infty} b_{n,-m} \zeta^{m}, \\
\Psi_{n}(g(\zeta))=-n b_{-n, 0}+n \sum_{m=1}^{\infty} b_{m,-n} \zeta^{-m}, \quad \Psi_{n}(f(\zeta))=\zeta^{-n}+n \sum_{m=1}^{\infty} b_{-n,-m} \zeta^{m} .
\end{array}
$$

The analysis above can be extended formally to the whole space $\tilde{\Sigma}$ and $\tilde{S}$. All the Taylor (Laurent) expansions are considered as formal power series expansions. All the identities hold formally. 


\section{Dispersionless hierarchies and tau funCtions}

We quickly review dispersionless Toda (dToda), dispersionless coupled modified KP (dcmKP) and dispersionless KP (dKP) hierarchies and their tau functions. For details, see 21, 22, 23, 24. For each of these dispersionless hierarchies, we give a new derivation of the dispersionless Hirota equation satisfied by the tau function, using the algebraic analysis we discussed in the previous section. We also prove that the dispersionless Hirota equations uniquely characterize the tau functions.

\subsection{Dispersionless Toda hierarchy.}

3.1.1. The hierarchy. The fundamental quantities in dToda hierarchy are two formal power series in $p$ :

$$
\begin{aligned}
\mathcal{L}(p) & =p+\sum_{n=0}^{\infty} u_{n+1}(t) p^{-n}, \\
\tilde{\mathcal{L}}^{-1}(p) & =\tilde{u}_{0}(t) p^{-1}+\sum_{n=0}^{\infty} \tilde{u}_{n+1}(t) p^{n} .
\end{aligned}
$$

Here $u_{n}(t)$ and $\tilde{u}_{n}(t)$ are functions of the independent variables $t_{n}, n \in \mathbb{Z}$, which we denote collectively by $t$. The Lax representation is ${ }^{3}$

$$
\begin{aligned}
\frac{\partial \mathcal{L}}{\partial t_{n}} & =\left\{\left(\mathcal{L}^{n}\right)_{\geq 0}, \mathcal{L}\right\}_{T}, & \frac{\partial \mathcal{L}}{\partial t_{-n}} & =\left\{\left(\tilde{\mathcal{L}}^{-n}\right)_{<0}, \mathcal{L}\right\}_{T}, \\
\frac{\partial \tilde{\mathcal{L}}}{\partial t_{n}} & =\left\{\left(\mathcal{L}^{n}\right)_{\geq 0}, \tilde{\mathcal{L}}\right\}_{T}, & \frac{\partial \tilde{\mathcal{L}}}{\partial t_{-n}} & =\left\{\left(\tilde{\mathcal{L}}^{-n}\right)_{<0}, \tilde{\mathcal{L}}\right\}_{T} .
\end{aligned}
$$

Here $\{\cdot, \cdot\}_{T}$ is the Poisson bracket for dToda hierarchy

$$
\{f, g\}_{T}=p \frac{\partial f}{\partial p} \frac{\partial g}{\partial t_{0}}-p \frac{\partial f}{\partial t_{0}} \frac{\partial g}{\partial p} .
$$

There exists a function $\phi(t)$ such that

$$
\frac{\partial \phi}{\partial t_{0}}=\log \tilde{u}_{0}, \quad \frac{\partial \phi}{\partial t_{n}}=\left(\mathcal{L}^{n}\right)_{0}, \quad \frac{\partial \phi}{\partial t_{-n}}=-\left(\tilde{\mathcal{L}}^{-n}\right)_{0}
$$

and two functions $\varphi=\sum_{n=1}^{\infty} \varphi_{n} p^{-n}, \psi=\sum_{n=1}^{\infty} \psi_{n} p^{n}$ such that

$$
\begin{aligned}
\mathcal{L} & =e^{\operatorname{ad} \varphi} p, & \tilde{\mathcal{L}} & =e^{\operatorname{ad} \tilde{\varphi}} p, \\
\nabla_{t_{n}, \varphi} \varphi & =-\left(\mathcal{L}^{n}\right)_{<0}, & \nabla_{t_{n}, \tilde{\varphi}} \tilde{\varphi} & =\left(\mathcal{L}^{n}\right)_{\geq 0}, \\
\nabla_{t_{-n}, \varphi} \varphi & =\left(\tilde{\mathcal{L}}^{-n}\right)_{<0} & \nabla_{t_{-n}, \tilde{\varphi}} \tilde{\varphi} & =-\left(\tilde{\mathcal{L}}^{-n}\right)_{\geq 0} .
\end{aligned}
$$

Here $(\operatorname{ad} f)(g)=\{f, g\}_{T}, \tilde{\varphi}$ is defined such that $e^{\operatorname{ad} \tilde{\varphi}}=e^{\operatorname{ad} \phi} e^{\operatorname{ad} \psi}$ and

$$
\nabla_{t, A} B=\sum_{n=0}^{\infty} \frac{(\operatorname{ad} A)^{n}}{(n+1) !} \frac{\partial B}{\partial t} .
$$

\footnotetext{
${ }^{3}$ Here it is understood that $p$ is a formal variable and does not depend on $t$.
} 
The Orlov-Schulman functions are defined by

$$
\begin{aligned}
& \mathcal{M}=e^{\operatorname{ad} \varphi}\left(\sum_{n=1}^{\infty} n t_{n} p^{n}+t_{0}\right)=\sum_{n=1}^{\infty} n t_{n} \mathcal{L}^{n}+t_{0}+\sum_{n=1}^{\infty} v_{n}^{T} \mathcal{L}^{-n} \\
& \tilde{\mathcal{M}}=e^{\operatorname{ad} \tilde{\varphi}}\left(-\sum_{n=1}^{\infty} n t_{-n} p^{-n}+t_{0}\right)=-\sum_{n=1}^{\infty} n t_{-n} \tilde{\mathcal{L}}^{-n}+t_{0}-\sum_{n=1}^{\infty} \tilde{v}_{n}^{T} \tilde{\mathcal{L}}^{n} .
\end{aligned}
$$

There exists a tau function $\tau_{\mathrm{d} \text { Toda }}$ such that

$$
\frac{\partial \log \tau_{\text {dToda }}}{\partial t_{0}}=\phi, \quad \frac{\partial \log \tau_{\text {dToda }}}{\partial t_{n}}=v_{n}^{T}, \quad \frac{\partial \log \tau_{\text {dToda }}}{\partial t_{-n}}=\tilde{v}_{n}^{T} .
$$

The tau function appears to be the dispersionless limit (leading term) of the tau function of a corresponding Toda hierarchy. The free energy of the hierarchy is defined by $\mathcal{F}=\log \tau_{\text {dToda }}$.

3.1.2. Dispersionless Hirota equation. The tau function generates the coefficients of $\mathcal{L}$. More precisely, we have the following identities:

$$
\begin{aligned}
\log p & =\log \mathcal{L}-\sum_{m=1}^{\infty} \frac{1}{m} \frac{\partial^{2} \mathcal{F}}{\partial t_{0} \partial t_{m}} \mathcal{L}^{-m} \\
\left(\mathcal{L}^{n}\right)_{\geq 0} & =\mathcal{L}^{n}-\sum_{m=1}^{\infty} \frac{1}{m} \frac{\partial^{2} \mathcal{F}}{\partial t_{n} \partial t_{m}} \mathcal{L}^{-m}=\frac{\partial^{2} \mathcal{F}}{\partial t_{0} \partial t_{n}}-\sum_{m=1}^{\infty} \frac{1}{m} \frac{\partial^{2} \mathcal{F}}{\partial t_{-m} \partial t_{n}} \tilde{\mathcal{L}}^{m} \\
\log p & =\log \tilde{\mathcal{L}}+\frac{\partial^{2} \mathcal{F}}{\partial t_{0}^{2}}-\sum_{m=1}^{\infty} \frac{1}{m} \frac{\partial^{2} \mathcal{F}}{\partial t_{-m} \partial t_{0}} \tilde{\mathcal{L}}^{m} \\
\left(\tilde{\mathcal{L}}^{-n}\right)_{<0} & =-\sum_{m=1}^{\infty} \frac{1}{m} \frac{\partial^{2} \mathcal{F}}{\partial t_{m} \partial t_{-n}} \mathcal{L}^{-m}=\tilde{\mathcal{L}}^{-n}+\frac{\partial^{2} \mathcal{F}}{\partial t_{0} \partial t_{-n}}-\sum_{m=1}^{\infty} \frac{1}{m} \frac{\partial^{2} \mathcal{F}}{\partial t_{-m} \partial t_{-n}} \tilde{\mathcal{L}}^{m}
\end{aligned}
$$

Now if we identify $p$ with $w, \mathcal{L}$ with $z$, then the first equation define $w$ as a function of $z$. Obviously, it belongs to the space $\Sigma$. We identify this function with our $w=g(z)$ in Section 2. In other words, $\mathcal{L}(p)$ is identified with the function $z=G(w)$, the inverse of $g$. Similarly, the third equation defines $w$ as a function of $\tilde{\mathcal{L}}$, which belongs to $\tilde{S}$. We identify this function with $w=f(z)$ or equivalently, $\tilde{\mathcal{L}}(p)$ is identified with $z=F(w)$, the inverse of $f$. Under these identifications, we see that the Faber polynomials $\Phi_{n}(w)$ 's are identified with $\left(\mathcal{L}^{n}(w)\right)_{\geq 0}$, and $\Psi_{n}(w)$ 's are identified with $\left(\tilde{\mathcal{L}}^{-n}(w)\right)_{\leq 0}$. Now compare (3.4) with (2.18), we find that the Grunsky coefficients $b_{n m}$ of the pair $(g=w(\mathcal{L}), f=w(\tilde{\mathcal{L}}))$ are related to the tau function or free energy 
by

$$
\begin{aligned}
b_{00} & =-\frac{\partial^{2} \mathcal{F}}{\partial t_{0}^{2}}, \quad b_{n, 0}=\frac{1}{n} \frac{\partial^{2} \mathcal{F}}{\partial t_{0} \partial t_{n}}, \quad b_{-n, 0}=\frac{1}{n} \frac{\partial^{2} \mathcal{F}}{\partial t_{0} \partial t_{-n}}, \quad n \geq 1, \\
b_{m, n} & =-\frac{1}{m n} \frac{\partial^{2} \mathcal{F}}{\partial t_{m} \partial t_{n}}, \quad b_{-m,-n}=-\frac{1}{m n} \frac{\partial^{2} \mathcal{F}}{\partial t_{-m} \partial t_{-n}}, \quad n, m \geq 1, \\
b_{-m, n} & =b_{n,-m}=-\frac{1}{m n} \frac{\partial^{2} \mathcal{F}}{\partial t_{-m} t_{n}}, \quad n, m \geq 1 .
\end{aligned}
$$

From (3.4), we can express $f$ and $g$ in terms of the tau function or free energy:

$$
g(z)=z \exp \left(-\sum_{m=1}^{\infty} \frac{1}{m} \frac{\partial^{2} \mathcal{F}}{\partial t_{0} \partial t_{m}} z^{-m}\right), \quad f(z)=z \exp \left(\frac{\partial^{2} \mathcal{F}}{\partial t_{0}^{2}}-\sum_{m=1}^{\infty} \frac{1}{m} \frac{\partial^{2} \mathcal{F}}{\partial t_{-m} \partial t_{0}} z^{m}\right) .
$$

As before, we define $g_{f}(z)=1 / f\left(z^{-1}\right)$, then

$$
g_{f}(z)=z \exp \left(-\frac{\partial^{2} \mathcal{F}}{\partial t_{0}^{2}}+\sum_{m=1}^{\infty} \frac{1}{m} \frac{\partial^{2} \mathcal{F}}{\partial t_{-m} \partial t_{0}} z^{-m}\right) .
$$

Hence rewriting the definition of the generalized Grunsky coefficients in terms of the tau function or free energy, we obtain the Hirota equation for dispersionless Toda hierarchy. Namely, from (2.6), (2.11), (2.12), we have

$$
\begin{aligned}
& z_{1} \exp \left(-\sum_{m=1}^{\infty} \frac{1}{m} \frac{\partial^{2} \mathcal{F}}{\partial t_{0} \partial t_{m}} z_{1}^{-m}\right)-z_{2} \exp \left(-\sum_{m=1}^{\infty} \frac{1}{m} \frac{\partial^{2} \mathcal{F}}{\partial t_{0} \partial t_{m}} z_{2}^{-m}\right) \\
= & \left(z_{1}-z_{2}\right) \exp \left(\sum_{m, n=1}^{\infty} \frac{1}{m n} \frac{\partial^{2} \mathcal{F}}{\partial t_{m} \partial t_{n}} z_{1}^{-m} z_{2}^{-n}\right), \\
& 1-\frac{1}{z_{1} z_{2}} \exp \left(\frac{\partial^{2} \mathcal{F}}{\partial t_{0}^{2}}+\sum_{m=1}^{\infty} \frac{1}{m} \frac{\partial^{2} \mathcal{F}}{\partial t_{0} \partial t_{m}} z_{1}^{-m}-\sum_{m=1}^{\infty} \frac{1}{m} \frac{\partial^{2} \mathcal{F}}{\partial t_{-m} \partial t_{0}} z_{2}^{-m}\right) \\
= & \exp \left(\sum_{m, n=1}^{\infty} \frac{1}{m n} \frac{\partial^{2} \mathcal{F}}{\partial t_{m} \partial t_{-n}} z_{1}^{-m} z_{2}^{-n}\right), \\
& z_{1} \exp \left(\sum_{m=1}^{\infty} \frac{1}{m} \frac{\partial^{2} \mathcal{F}}{\partial t_{-m} \partial t_{0}} z_{1}^{-m}\right)-z_{2} \exp \left(\sum_{m=1}^{\infty} \frac{1}{m} \frac{\partial^{2} \mathcal{F}}{\partial t_{-m} \partial t_{0}} z_{2}^{-m}\right) \\
= & \left(z_{1}-z_{2}\right) \exp \left(\sum_{m, n=1}^{\infty} \frac{1}{m n} \frac{\partial^{2} \mathcal{F}}{\partial t_{-m} \partial t_{-n}} z_{1}^{-m} z_{2}^{-n}\right) .
\end{aligned}
$$

We should understand these identities as defining a sequence of relations satisfied by the second derivatives of $\mathcal{F}$ by comparing the coefficients of $z_{1}^{-m} z_{2}^{-n}$ on both sides. 
Conversely, the tau function is uniquely characterized by these Hirota equations.

Proposition 3.1. If $\mathcal{F}=\log \tau$ is a function of $t_{n}, n \in \mathbb{Z}$ that satisfies the Hirota equations (3.6), then $\tau$ is a tau function of a solution of the dToda hierarchy. More explicitly, if we define $\mathcal{L}(p)$ and $\tilde{\mathcal{L}}(p)$ by formally inverting the functions $\mathfrak{p}(\mathcal{L})$ and $\tilde{\mathfrak{p}}(\tilde{\mathcal{L}})$ defined by

$$
\begin{aligned}
& \log \mathfrak{p}(\mathcal{L})=\log \mathcal{L}-\sum_{m=1}^{\infty} \frac{1}{m} \frac{\partial^{2} \mathcal{F}}{\partial t_{0} \partial t_{m}} \mathcal{L}^{-m} \\
& \log \tilde{\mathfrak{p}}(\tilde{\mathcal{L}})=\log \tilde{\mathcal{L}}+\frac{\partial^{2} \mathcal{F}}{\partial t_{0}^{2}}-\sum_{m=1}^{\infty} \frac{1}{m} \frac{\partial^{2} \mathcal{F}}{\partial t_{-m} \partial t_{0}} \tilde{\mathcal{L}}^{m}
\end{aligned}
$$

then $(\mathcal{L}, \tilde{\mathcal{L}})$ satisfies the Lax equations (3.1) ${ }^{4}$ for dToda hierarchy.

Proof. In the first part of the proof, we trace the reasoning above backward. We define the function $g \in \Sigma$ and $f \in \tilde{S}$ by

$\log \frac{g(z)}{z}=-\sum_{m=1}^{\infty} \frac{1}{m} \frac{\partial^{2} \mathcal{F}}{\partial t_{0} \partial t_{m}} z^{-m}, \quad \log \frac{f(z)}{z}=\frac{\partial^{2} \mathcal{F}}{\partial t_{0}^{2}}-\sum_{m=1}^{\infty} \frac{1}{m} \frac{\partial^{2} \mathcal{F}}{\partial t_{-m} \partial t_{0}} z^{m}$.

We define the generalized Grunsky coefficients of the pair $(f, g)$ by the equations (2.6), (2.7), (2.8). We also define $g_{f}(z)=1 / f\left(z^{-1}\right)$, then

$$
\log \frac{g_{f}(z)}{z}=-\frac{\partial^{2} \mathcal{F}}{\partial t_{0}^{2}}+\sum_{m=1}^{\infty} \frac{1}{m} \frac{\partial^{2} \mathcal{F}}{\partial t_{-m} \partial t_{0}} z^{-m} .
$$

In terms of $g$ and $g_{f}$, the Hirota equations (3.6) read as

$$
\begin{aligned}
\log \frac{g\left(z_{1}\right)-g\left(z_{2}\right)}{z_{1}-z_{2}} & =\sum_{m, n=1}^{\infty} \frac{1}{m n} \frac{\partial^{2} \mathcal{F}}{\partial t_{m} \partial t_{n}} z_{1}^{-m} z_{2}^{-n}, \\
\log \left(1-\frac{1}{g\left(z_{1}\right) g_{f}\left(z_{2}\right)}\right) & =\sum_{m, n=1}^{\infty} \frac{1}{m n} \frac{\partial^{2} \mathcal{F}}{\partial t_{m} \partial t_{-n}} z_{1}^{-m} z_{2}^{-n}, \\
\log \frac{g_{f}\left(z_{1}\right)-g_{f}\left(z_{2}\right)}{z_{1}-z_{2}} & =-\frac{\partial^{2} \mathcal{F}}{\partial t_{0}^{2}}+\sum_{m, n=1}^{\infty} \frac{1}{m n} \frac{\partial^{2} \mathcal{F}}{\partial t_{-m} \partial t_{-n}} z_{1}^{-m} z_{2}^{-n} .
\end{aligned}
$$

Comparing with (2.6), (2.9), (2.11), (2.12), we find the Grunsky coefficients in terms of $\mathcal{F}$ are given by the equations in (3.5). Hence if we define $\mathcal{L}(p)$ to be the inverse function of $w=g(z)$, and $\tilde{\mathcal{L}}(p)$ to be the inverse function

\footnotetext{
${ }^{4}$ The coefficients of the functions $\mathfrak{p}$ and $\tilde{\mathfrak{p}}$ depend on $t$ since they can be expressed as functions of second derivatives of $\mathcal{F}$ by exponentiating (3.7). The dependence of the coefficients of $\mathcal{L}$ and $\tilde{\mathcal{L}}$ on $t$ are understood in the following way: They are inverses of $\mathfrak{p}$ and $\tilde{\mathfrak{p}}$ respectively. Their coefficients can be solved in terms of the coefficients of $\mathfrak{p}$ and $\tilde{\mathfrak{p}}$ and are hence functions of $t$.
} 
of $w=f(z)$ by replacing $w$ with $p$, then the identities satisfied by the Faber polynomials of $f$ and $g$ (2.18) say that

$$
\begin{aligned}
& \Phi_{n}=\left(\mathcal{L}^{n}\right)_{\geq 0}=\mathcal{L}^{n}-\sum_{m=1}^{\infty} \frac{1}{m} \frac{\partial^{2} \mathcal{F}}{\partial t_{n} \partial t_{m}} \mathcal{L}^{-m}=\frac{\partial^{2} \mathcal{F}}{\partial t_{0} \partial t_{n}}-\sum_{m=1}^{\infty} \frac{1}{m} \frac{\partial^{2} \mathcal{F}}{\partial t_{-m} \partial t_{n}} \tilde{\mathcal{L}}^{m}, \\
& \Psi_{n}=\left(\tilde{\mathcal{L}}^{-n}\right)_{\leq 0}=-\frac{\partial^{2} \mathcal{F}}{\partial t_{0} \partial t_{-n}}-\sum_{m=1}^{\infty} \frac{1}{m} \frac{\partial^{2} \mathcal{F}}{\partial t_{m} \partial t_{-n}} \mathcal{L}^{-m}=\tilde{\mathcal{L}}^{-n}-\sum_{m=1}^{\infty} \frac{1}{m} \frac{\partial^{2} \mathcal{F}}{\partial t_{-m} \partial t_{-n}} \tilde{\mathcal{L}}^{m} .
\end{aligned}
$$

Comparing the coefficients of $p^{0}$ term in the last equation, we have

$$
\left(\tilde{\mathcal{L}}^{-n}\right)_{0}=-\frac{\partial^{2} \mathcal{F}}{\partial t_{0} \partial t_{-n}} .
$$

Hence we can rewrite the last equation as

$$
\left(\tilde{\mathcal{L}}^{-n}\right)_{<0}=-\sum_{m=1}^{\infty} \frac{1}{m} \frac{\partial^{2} \mathcal{F}}{\partial t_{m} \partial t_{-n}} \mathcal{L}^{-m}=\tilde{\mathcal{L}}^{-n}+\frac{\partial^{2} \mathcal{F}}{\partial t_{0} \partial t_{-n}}-\sum_{m=1}^{\infty} \frac{1}{m} \frac{\partial^{2} \mathcal{F}}{\partial t_{-m} \partial t_{-n}} \tilde{\mathcal{L}}^{m} .
$$

Now from (3.7), (3.8) and (3.9), we have

$$
\begin{aligned}
& \left.\frac{1}{\mathfrak{p}(\mathcal{L})} \frac{\partial \mathfrak{p}(\mathcal{L})}{\partial t_{n}}\right|_{\mathcal{L} \text { fixed }}=-\sum_{m=1}^{\infty} \frac{1}{m} \frac{\partial^{3} \mathcal{F}}{\partial t_{n} \partial t_{0} \partial t_{m}} \mathcal{L}^{-m}=\left.\frac{\partial\left(\mathcal{L}^{n}\right)_{\geq 0}}{\partial t_{0}}\right|_{\mathcal{L} \text { fixed }}, \\
& \left.\frac{1}{\mathfrak{p}(\mathcal{L})} \frac{\partial \mathfrak{p}(\mathcal{L})}{\partial t_{-n}}\right|_{\mathcal{L} \text { fixed }}=-\sum_{m=1}^{\infty} \frac{1}{m} \frac{\partial^{3} \mathcal{F}}{\partial t_{-n} \partial t_{0} \partial t_{m}} \mathcal{L}^{-m}=\left.\frac{\partial\left(\tilde{\mathcal{L}}^{-n}\right)_{<0}}{\partial t_{0}}\right|_{\mathcal{L} \text { fixed }}, \\
& \left.\frac{1}{\tilde{\mathfrak{p}}(\tilde{\mathcal{L}})} \frac{\partial \tilde{\mathfrak{p}}(\tilde{\mathcal{L}})}{\partial t_{n}}\right|_{\tilde{\mathcal{L}} \text { fixed }}=\frac{\partial^{3} \mathcal{F}}{\partial t_{n} \partial t_{0}^{2}}-\sum_{m=1}^{\infty} \frac{1}{m} \frac{\partial^{3} \mathcal{F}}{\partial t_{n} \partial t_{0} \partial t_{-m}} \tilde{\mathcal{L}}^{m}=\left.\frac{\partial\left(\mathcal{L}^{n}\right)_{\geq 0}}{\partial t_{0}}\right|_{\tilde{\mathcal{L}} \text { fixed }}, \\
& \left.\frac{1}{\tilde{\mathfrak{p}}(\tilde{\mathcal{L}})} \frac{\partial \tilde{\mathfrak{p}}(\tilde{\mathcal{L}})}{\partial t_{-n}}\right|_{\tilde{\mathcal{L}} \text { fixed }}=\frac{\partial^{3} \mathcal{F}}{\partial t_{-n} \partial t_{0}^{2}}-\sum_{m=1}^{\infty} \frac{1}{m} \frac{\partial^{3} \mathcal{F}}{\partial t_{-n} \partial t_{0} \partial t_{-m}} \tilde{\mathcal{L}}^{m}=\left.\frac{\partial\left(\tilde{\mathcal{L}}^{-n}\right)_{<0}}{\partial t_{0}}\right|_{\tilde{\mathcal{L}} \text { fixed }} .
\end{aligned}
$$

On the other hand, since $\mathfrak{p} \circ \mathcal{L}$ is the identity function in $p$, by chain rule, we have

$$
\frac{\partial \mathfrak{p}(\mathcal{L})}{\partial \mathcal{L}} \frac{\partial \mathcal{L}}{\partial t}+\left.\frac{\partial \mathfrak{p}(\mathcal{L})}{\partial t}\right|_{\mathcal{L} \text { fixed }}=0
$$

and similarly for $\tilde{\mathcal{L}}$. Here $t$ is any of the independent variables. Hence

$$
\begin{aligned}
\frac{\partial \mathcal{L}}{\partial t_{n}} & =-\left.\frac{\partial \mathcal{L}}{\partial p} \frac{\partial \mathfrak{p}(\mathcal{L})}{\partial t_{n}}\right|_{\mathcal{L} \text { fixed }}=-\left.p \frac{\partial \mathcal{L}}{\partial p} \frac{\partial\left(\mathcal{L}^{n}\right)_{\geq 0}}{\partial t_{0}}\right|_{\mathcal{L} \text { fixed }} \\
& =-p \frac{\partial \mathcal{L}}{\partial p}\left(\frac{\partial\left(\mathcal{L}^{n}\right)_{\geq 0}}{\partial t_{0}}-\frac{\partial\left(\mathcal{L}^{n}\right)_{\geq 0}}{\partial \mathcal{L}} \frac{\partial \mathcal{L}}{\partial t_{0}}\right)=p\left(\frac{\partial\left(\mathcal{L}^{n}\right)_{\geq 0}}{\partial p} \frac{\partial \mathcal{L}}{\partial t_{0}}-\frac{\partial \mathcal{L}}{\partial p} \frac{\partial\left(\mathcal{L}^{n}\right)_{\geq 0}}{\partial t_{0}}\right) \\
& =\left\{\left(\mathcal{L}^{n}\right)_{\geq 0}, \mathcal{L}\right\}_{T},
\end{aligned}
$$


which is the first one of the Lax equations (3.1). The other equations are derived in the same way from (3.10).

Remark 3.2. The equations in (3.10) are sometimes taken to be the definition of dispersionless Toda hierarchy.

There are two variants of the dToda hierarchy that we would like to discuss here. Let $(\mathcal{L}, \tilde{\mathcal{L}})$ be a solution of the dToda hierarchy and $\phi$ be the function defined by (3.2), then we can make a Miura-type transformation (Lemma 2.1.3 in [23] ) and define $\mathcal{L}^{\prime}=e^{-\operatorname{ad} \phi} \mathcal{L}, \tilde{\mathcal{L}}^{\prime}=e^{-\operatorname{ad} \phi} \tilde{\mathcal{L}}$. Then they are of the form

$$
\begin{gathered}
\mathcal{L}^{\prime}(p)=\tilde{u}_{0} p+\sum_{n=0}^{\infty} u_{n+1}^{\prime}(t) p^{-n}=\tilde{u}_{0} p+\sum_{n=0}^{\infty} u_{n+1}(t)\left(\tilde{u_{0}} p\right)^{-n}, \\
\tilde{\mathcal{L}}^{\prime-1}(p)=p^{-1}+\sum_{n=0}^{\infty} \tilde{u}_{n+1}^{\prime}(t) p^{n}=p^{-1}+\sum_{n=0}^{\infty} \tilde{u}_{n+1}(t)\left(\tilde{u}_{0} p\right)^{n}
\end{gathered}
$$

and satisfy the Lax equations

$$
\begin{aligned}
\frac{\partial \mathcal{L}^{\prime}}{\partial t_{n}} & =\left\{\left(\left(\mathcal{L}^{\prime}\right)^{n}\right)_{>0}, \mathcal{L}^{\prime}\right\}_{T}, & \frac{\partial \mathcal{L}^{\prime}}{\partial t_{-n}} & \left.=\left\{\left(\left(\tilde{\mathcal{L}}^{\prime}\right)^{-n}\right)\right)_{\leq 0}, \mathcal{L}^{\prime}\right\}_{T}, \\
\frac{\partial \tilde{\mathcal{L}}^{\prime}}{\partial t_{n}} & =\left\{\left(\left(\mathcal{L}^{\prime}\right)^{n}\right)_{>0}, \tilde{\mathcal{L}}^{\prime}\right\}_{T}, & \frac{\partial \tilde{\mathcal{L}}^{\prime}}{\partial t_{-n}} & =\left\{\left(\left(\tilde{\mathcal{L}}^{\prime}\right)^{-n}\right)_{\leq 0}, \tilde{\mathcal{L}}^{\prime}\right\}_{T} .
\end{aligned}
$$

Now the dressing functions for $\mathcal{L}^{\prime}$ and $\tilde{\mathcal{L}}^{\prime}$ are $\varphi^{\prime}$ and $\tilde{\varphi}^{\prime}$ respectively, where $e^{\operatorname{ad} \varphi^{\prime}}=e^{-\operatorname{ad} \phi} e^{\operatorname{ad} \varphi}$ and $e^{\operatorname{ad} \tilde{\varphi}^{\prime}}=e^{-\operatorname{ad} \phi} e^{\operatorname{ad} \tilde{\varphi}}$. The Orlov-Schulman functions $\mathcal{M}^{\prime}$ and $\tilde{\mathcal{M}}^{\prime}$ are defined as in (3.3), and we find that

$$
\begin{aligned}
& \mathcal{M}^{\prime}=e^{-\operatorname{ad} \phi} \mathcal{M}=\sum_{n=1}^{\infty} n t_{n}\left(\mathcal{L}^{\prime}\right)^{n}+t_{0}+\sum_{n=1}^{\infty} v_{n}^{T}\left(\mathcal{L}^{\prime}\right)^{-n}, \\
& \tilde{\mathcal{M}}^{\prime}=e^{-\operatorname{ad} \phi} \tilde{\mathcal{M}}=-\sum_{n=1}^{\infty} n t_{-n}\left(\tilde{\mathcal{L}}^{\prime}\right)^{-n}+t_{0}-\sum_{n=1}^{\infty} \tilde{v}_{n}^{T}\left(\tilde{\mathcal{L}}^{\prime}\right)^{n} .
\end{aligned}
$$

In particular, the functions $v_{n}^{T}$ 's and $\tilde{v}_{n}^{T}$ 's are not changed. Hence the tau function is the same. If we look at this transformation from the point of view of conformal maps, what it amounts to is the pre-composition of $z=G(w)$ and $z=F(w)$ with the linear map $w \mapsto \tilde{u_{0}} w\left(\tilde{u}_{0}=a_{1}\right.$, the leading coefficient of $f(z)$ ). Hence for the inverse function, we have

$$
g^{\prime}(z)=\frac{g(z)}{\tilde{u}_{0}}, \quad f^{\prime}(z)=\frac{f(z)}{\tilde{u}_{0}} .
$$

From these and the definition of the Grunsky coefficients, it is quite obvious that the Grunsky coefficients are not changed. In terms of the tau function, 
we now have

$$
\begin{aligned}
& \mathfrak{p}\left(\mathcal{L}^{\prime}\right)=g^{\prime}\left(\mathcal{L}^{\prime}\right)=\mathcal{L}^{\prime} \exp \left(-\frac{\partial^{2} \mathcal{F}}{\partial t_{0}^{2}}-\sum_{m=1}^{\infty} \frac{1}{m} \frac{\partial^{2} \mathcal{F}}{\partial t_{0} \partial t_{m}}\left(\mathcal{L}^{\prime}\right)^{-m}\right), \\
& \tilde{\mathfrak{p}}\left(\tilde{\mathcal{L}}^{\prime}\right)=f^{\prime}\left(\tilde{\mathcal{L}}^{\prime}\right)=\tilde{\mathcal{L}}^{\prime} \exp \left(-\sum_{m=1}^{\infty} \frac{1}{m} \frac{\partial^{2} \mathcal{F}}{\partial t_{-m} \partial t_{0}}\left(\tilde{\mathcal{L}}^{\prime}\right)^{m}\right)
\end{aligned}
$$

and the dispersionless Hirota equations for $\left(\mathcal{L}^{\prime}, \tilde{\mathcal{L}}^{\prime}\right)$ assume the same form (3.6) .

Another variant of the dToda hierarchy is the one favored by Wiegmann and Zabrodin in association with conformal maps [25, 16, 13. Now the Miura type transformation is defined as $\mathcal{L}^{\prime}=e^{-\operatorname{ad} \phi / 2} \mathcal{L}, \tilde{\mathcal{L}}^{\prime}=e^{-\operatorname{ad} \phi / 2} \tilde{\mathcal{L}}$. Then they are of the form

$$
\begin{gathered}
\mathcal{L}^{\prime}(p)=r p+\sum_{n=0}^{\infty} u_{n+1}^{\prime}(t) p^{-n}=r p+\sum_{n=0}^{\infty} u_{n+1}(t)(r p)^{-n} \\
\tilde{\mathcal{L}}^{\prime-1}(p)=r p^{-1}+\sum_{n=0}^{\infty} \tilde{u}_{n+1}^{\prime}(t) p^{n}=r p^{-1}+\sum_{n=0}^{\infty} \tilde{u}_{n+1}(t)(r p)^{n},
\end{gathered}
$$

where $r$ is a square root of $\tilde{u}_{0}$. The Lax equations become ${ }^{5}$

$$
\begin{array}{rlrl}
\frac{\partial \mathcal{L}^{\prime}}{\partial t_{n}} & =\left\{\mathcal{H}_{n}, \mathcal{L}^{\prime}\right\}_{T}, & & \frac{\partial \mathcal{L}^{\prime}}{\partial t_{-n}}=\left\{\tilde{\mathcal{H}}_{n}, \mathcal{L}^{\prime}\right\}_{T}, \\
\frac{\partial \tilde{\mathcal{L}}^{\prime}}{\partial t_{n}} & =\left\{\mathcal{H}_{n}, \tilde{\mathcal{L}}^{\prime}\right\}_{T}, & \frac{\partial \tilde{\mathcal{L}}^{\prime}}{\partial t_{-n}}=\left\{\tilde{\mathcal{H}}_{n}, \tilde{\mathcal{L}}^{\prime}\right\}_{T} \\
\mathcal{H}_{n}=\left(\left(\mathcal{L}^{\prime}\right)^{n}\right)_{>0}+\frac{1}{2}\left(\left(\mathcal{L}^{\prime}\right)^{n}\right)_{0}, & \tilde{\mathcal{H}}_{n}=\left(\left(\tilde{\mathcal{L}}^{\prime}\right)^{-n}\right)_{<0}+\frac{1}{2}\left(\left(\tilde{\mathcal{L}}^{\prime}\right)^{-n}\right)_{0} .
\end{array}
$$

This version has the advantage that the roles of $\mathcal{L}$ and $\mathcal{L}^{\prime}$ are symmetric. The same discussion above shows that the tau function and dispersionless Hirota equations assume the same form but now

$$
\begin{aligned}
& \mathfrak{p}\left(\mathcal{L}^{\prime}\right)=g^{\prime}\left(\mathcal{L}^{\prime}\right)=\mathcal{L}^{\prime} \exp \left(-\frac{1}{2} \frac{\partial^{2} \mathcal{F}}{\partial t_{0}^{2}}-\sum_{m=1}^{\infty} \frac{1}{m} \frac{\partial^{2} \mathcal{F}}{\partial t_{0} \partial t_{m}}\left(\mathcal{L}^{\prime}\right)^{-m}\right), \\
& \tilde{\mathfrak{p}}\left(\tilde{\mathcal{L}}^{\prime}\right)=f^{\prime}\left(\tilde{\mathcal{L}}^{\prime}\right)=\tilde{\mathcal{L}}^{\prime} \exp \left(\frac{1}{2} \frac{\partial^{2} \mathcal{F}}{\partial t_{0}^{2}}-\sum_{m=1}^{\infty} \frac{1}{m} \frac{\partial^{2} \mathcal{F}}{\partial t_{-m} \partial t_{0}}\left(\tilde{\mathcal{L}}^{\prime}\right)^{m}\right) .
\end{aligned}
$$

We can also view the dispersionless Hirota equations as a consequence of the definition of the Grunsky coefficients for the pair $\left(g, g_{f}\right)$. From this point of view and our discussion above, we readily see that if $(\mathcal{L}, \tilde{\mathcal{L}})$ is a solution to the first version of the dToda hierarchy, then the pair $\left(\mathcal{L}^{\prime}, \tilde{\mathcal{L}}^{\prime}\right)$, where $\mathcal{L}^{\prime}(p)=\tilde{\mathcal{L}}(1 / p)^{-1}, \tilde{\mathcal{L}}^{\prime}(p)=\mathcal{L}(1 / p)^{-1}$ is a solution to the second version of the dToda hierarchy, if we redefine the independent variables as $t_{n}^{\prime}=t_{-n}$ and $t_{-n}^{\prime}=t_{n}$.

\footnotetext{
${ }^{5}$ Notice that the $\bar{t}_{n}$ in [25] is the complex conjugate of $t_{n}$. It amounts to $-t_{-n}$ here.
} 
3.2. Dispersionless (coupled) modified KP hierarchy. We define the dispersionless coupled modified KP hierarchy (dcmKP) in [24]. Here we are only interested in a special case. (In the notation in [24, it is the case where $\mathcal{P}=k$.)

3.2.1. The hierarchy. The fundamental quantity is a formal power series

$$
\mathcal{L}=k+\sum_{n=0}^{\infty} u_{n+1}(t) k^{-n}
$$

with coefficients depending on the parameters $t=\left(x, t_{0}, t_{1}, t_{2} \ldots\right)$. The Lax representation of the dcmKP in our special case here is

$$
\frac{\partial \mathcal{L}}{\partial t_{n}}=\left\{\left(\mathcal{L}^{n}\right)_{>0}, \mathcal{L}\right\}, \quad n \geq 1 ; \quad \frac{\partial \mathcal{L}}{\partial t_{0}}=\frac{1}{k} \frac{\partial \mathcal{L}}{\partial x}
$$

Here $\{\cdot, \cdot\}$ is the Poisson bracket defined as $\{f, g\}=\frac{\partial f}{\partial k} \frac{\partial g}{\partial x}-\frac{\partial f}{\partial x} \frac{\partial g}{\partial k}$. The $n=1$ flow of the hierarchy says that

$$
\frac{\partial \mathcal{L}}{\partial t_{1}}=\frac{\partial \mathcal{L}}{\partial x}
$$

Hence all the dependence on $t_{1}$ and $x$ appears in the form $t_{1}+x$. There exists a dressing function $\varphi=\sum_{n=0}^{\infty} \varphi_{n}(t) k^{-n}$ and a function $\phi(t)$ such that

$$
\begin{array}{rlrl}
\mathcal{L} & =e^{\operatorname{ad} \varphi} k, & & \frac{\partial \phi}{\partial t_{n}}=\left(\mathcal{L}^{n}\right)_{0}, n \geq 1, \\
\nabla_{t_{n}, \varphi} \varphi & =-\left(\mathcal{L}^{n}\right)_{\leq 0}, \quad n \geq 1 ; & \nabla_{t_{0}, \varphi} \varphi=\log k-\log \mathcal{L} .
\end{array}
$$

Here $(\operatorname{ad} f)(g)=\{f, g\}$. The Orlov-Schulman function is defined as

$\mathcal{M}=e^{\operatorname{ad} \varphi}\left(\sum_{n=1}^{\infty} n t_{n} k^{n-1}+x+\frac{t_{0}}{k}\right)=\sum_{n=1}^{\infty} n t_{n} \mathcal{L}^{n-1}+x+\frac{t_{0}}{\mathcal{L}}+\sum_{n=1}^{\infty} v_{n} \mathcal{L}^{-n-1}$.

There exists a tau function $\tau$ such that

$$
\frac{\partial \log \tau}{\partial t_{n}}=v_{n}, \quad n \geq 1, \quad \frac{\partial \log \tau}{\partial t_{0}}=\phi .
$$

We define the free energy of the hierarchy by $\mathcal{F}=\log \tau$.

3.2.2. Dispersionless Hirota equation. In terms of the tau function or the free energy, we have the following identities:

$$
\begin{aligned}
\log k & =\log \mathcal{L}-\sum_{m=1}^{\infty} \frac{1}{m} \frac{\partial^{2} \mathcal{F}}{\partial t_{m} \partial t_{0}} \mathcal{L}^{-m}, \\
\left(\mathcal{L}^{n}\right)_{>0} & =\mathcal{L}^{n}-\frac{\partial^{2} \mathcal{F}}{\partial t_{0} \partial t_{n}}-\sum_{m=1}^{\infty} \frac{1}{m} \frac{\partial^{2} \mathcal{F}}{\partial t_{n} \partial t_{m}} \mathcal{L}^{-m} .
\end{aligned}
$$

From the second equation, we also have

$$
\left(\mathcal{L}^{n}\right)_{\geq 0}=\mathcal{L}^{n}-\sum_{m=1}^{\infty} \frac{1}{m} \frac{\partial^{2} \mathcal{F}}{\partial t_{n} \partial t_{m}} \mathcal{L}^{-m}
$$


Now we identify $k$ with $w$ and $\mathcal{L}$ with $z$. The first equation in (3.14) defines $w$ as a function of $z$, which we denote by $g(z)$ :

$$
g(z)=z \exp \left(-\sum_{m=1}^{\infty} \frac{1}{m} \frac{\partial^{2} \mathcal{F}}{\partial t_{m} \partial t_{0}} z^{-m}\right) .
$$

Obviously, $g \in \Sigma$. The Faber polynomials $\Phi_{n}$ for $g$ are then identified with $\left(\mathcal{L}^{n}\right)_{\geq 0}$. Comparing (2.4) to (3.15), we find that the Grunsky coefficients of $g(z)$ are related to the tau function or the free energy by

$$
b_{m n}=-\frac{1}{m n} \frac{\partial^{2} \mathcal{F}}{\partial t_{m} \partial t_{n}} .
$$

Together with (3.16), we can rewrite the definition of the Grunsky coefficients (2.3) as

$$
\begin{aligned}
& z_{1} \exp \left(-\sum_{m=1}^{\infty} \frac{1}{m} \frac{\partial^{2} \mathcal{F}}{\partial t_{0} \partial t_{m}} z_{1}^{-m}\right)-z_{2} \exp \left(-\sum_{m=1}^{\infty} \frac{1}{m} \frac{\partial^{2} \mathcal{F}}{\partial t_{0} \partial t_{m}} z_{2}^{-m}\right) \\
= & \left(z_{1}-z_{2}\right) \exp \left(\sum_{m, n=1}^{\infty} \frac{1}{m n} \frac{\partial^{2} \mathcal{F}}{\partial t_{m} \partial t_{n}} z_{1}^{-m} z_{2}^{-n}\right),
\end{aligned}
$$

which is the dispersionless Hirota equation for this special case of dcmKP hierarchy.

Conversely, we can characterize the tau function for dcmKP hierarchy as:

Proposition 3.3. If $\mathcal{F}=\log \tau$ is a function of $t_{n}, n \geq 0$ that satisfies the Hirota equation (3.18) and

$$
\frac{\partial^{3} \log \tau}{\partial t_{0}^{2} \partial t_{1}}=0
$$

then $\tau$ is a tau function of a solution of the dcmKP hierarchy. More explicitly, if we define $\mathcal{L}(k)$ by formally inverting the function $\mathrm{k}(\mathcal{L})$ defined by

$$
\log \mathrm{k}(\mathcal{L})=\log \mathcal{L}-\sum_{m=1}^{\infty} \frac{1}{m} \frac{\partial^{2} \mathcal{F}}{\partial t_{0} \partial t_{m}} \mathcal{L}^{-m},
$$

and replacing $t_{1}$ by $t_{1}+x$, then $\mathcal{L}$ satisfies the Lax equations (3.12) for dcmKP hierarchy.

Proof. The proof is almost the same as the case of dToda. Identifying $\mathcal{L}$ with $z$, we define the function $g(z) \in \Sigma$ by

$$
\log g(z)=\log z-\sum_{m=1}^{\infty} \frac{1}{m} \frac{\partial^{2} \mathcal{F}}{\partial t_{0} \partial t_{m}} z^{-m} .
$$


The dispersionless Hirota equation (3.18) then read as

$$
\log \frac{g\left(z_{1}\right)-g\left(z_{2}\right)}{z_{1}-z_{2}}=\sum_{m, n=1}^{\infty} \frac{1}{m n} \frac{\partial^{2} \mathcal{F}}{\partial t_{m} \partial t_{n}} z_{1}^{-m} z_{2}^{-n}
$$

Comparing with the definition of the Grunsky coefficients of $g$ given by equation (2.3), we find that the relation between the Grunsky coefficients and free energy is given by (3.17). Let $z=G(w)$ be the inverse of $g(z)$. Then $G(w)$ is the function $\mathcal{L}(k)$ defined by (3.20) if we identify $k$ with $w$. The Faber polynomials of $g(z)$ is then identified with $(\mathcal{L}(w))_{\geq 0}$. We can then rewrite the identity satisfied by the Faber polynomials (2.4) in terms of the free energy by

$$
\left(\mathcal{L}^{n}\right)_{\geq 0}=\mathcal{L}^{n}-\sum_{m=1}^{\infty} \frac{1}{m} \frac{\partial^{2} \mathcal{F}}{\partial t_{m} \partial t_{n}} \mathcal{L}^{-m}
$$

From (3.20), we also have

Hence

$$
\frac{\partial^{2} \mathcal{F}}{\partial t_{n} \partial t_{0}}=\operatorname{Res} \mathcal{L}^{n} d \log k=\left(\mathcal{L}^{n}\right)_{0}
$$

From (3.20) and the $n=1$ case in (3.21), we have

$$
\left.\frac{1}{\mathrm{k}} \frac{\partial \mathrm{k}}{\partial t_{1}}\right|_{\mathcal{L} \text { fixed }}=-\sum_{m=1}^{\infty} \frac{1}{m} \frac{\partial^{3} \mathcal{F}}{\partial t_{m} \partial t_{0} \partial t_{1}}=\left.\frac{\partial \mathrm{k}}{\partial t_{0}}\right|_{\mathcal{L} \text { fixed }}
$$

We have used $(\mathcal{L})_{>0}=k$ and (3.19). Using the identity (3.11) (with $p$ replaced by $k$ ), it follows that

$$
\frac{\partial \mathcal{L}}{\partial x}=\frac{\partial \mathcal{L}}{\partial t_{1}}=k \frac{\partial \mathcal{L}}{\partial t_{0}}
$$

This gives the second equation in the Lax equations (3.12). Using the $n=1$ case in (3.21) and $(\mathcal{L})_{>0}=k$ again, we have

$$
\left.\frac{\partial k}{\partial t_{n}}\right|_{\mathcal{L} \text { fixed }}=-\frac{\partial^{2} \mathcal{F}}{\partial t_{1} \partial t_{n} \partial t_{0}}-\sum_{m=1}^{\infty} \frac{1}{m} \frac{\partial^{2} \mathcal{F}}{\partial t_{m} \partial t_{n} \partial t_{1}} \mathcal{L}^{-m}=\left.\frac{\partial\left(\mathcal{L}^{n}\right)_{>0}}{\partial t_{1}}\right|_{\mathcal{L} \text { fixed }},
$$

which by (3.11) is equivalent to

$$
\begin{aligned}
\frac{\partial \mathcal{L}}{\partial t_{n}} & =-\left.\frac{\partial \mathcal{L}}{\partial k} \frac{\partial\left(\mathcal{L}^{n}\right)_{>0}}{\partial t_{1}}\right|_{\mathcal{L} \text { fixed }}=-\frac{\partial \mathcal{L}}{\partial k}\left(\frac{\partial\left(\mathcal{L}^{n}\right)_{>0}}{\partial x}-\frac{\partial\left(\mathcal{L}^{n}\right)_{>0}}{\partial \mathcal{L}} \frac{\partial \mathcal{L}}{\partial x}\right) \\
& =\left\{\left(\mathcal{L}^{n}\right)_{>0}, \mathcal{L}\right\}
\end{aligned}
$$

Compare the dispersionless Hirota equations for dToda and dcmKP hierarchies, we immediately have 
Corollary 3.4. If $(\mathcal{L}, \tilde{\mathcal{L}})$ is a solution to the dToda hierarchy (3.1), and $\frac{\partial(\mathcal{L})_{0}}{\partial t_{0}}=0$, then $\mathcal{L}$ is a solution to the dcmKP hierarchy (3.12), when we replace $t_{1}$ by $t_{1}+x$ and regard the $t_{-n}$ 's, $n \geq 1$ as parameters. The tau function for the dToda hierarchy is the tau function for the corresponding dcmKP hierarchy.

Proof. In [24, we proved this proposition by comparing the Lax equations. Here we just notice that the first equation in the Hirota equations for dToda (3.6) is identical with the Hirota equation for dcmKP (3.18). The result follows from the proposition above.

3.3. Dispersionless KP hierarchy. . This is the most well known case. The dispersionless Hirota equation for dKP hierarchy was first derived as the quasi-classical limit of the differential Fay identity by Takasaki and Takebe in [23], see also the work of Carroll and Kodama [5]. Here we derive the Hirota equation along the same line as we do for dToda and dcmKP hierarchies.

3.3.1. The hierarchy. The fundamental quantity in dKP hierarchy is a formal power series

$$
\mathcal{L}=k+\sum_{n=1}^{\infty} u_{n+1}(t) k^{-n}
$$

with coefficients depending on the independent variables $t=\left(x, t_{1}, t_{2}, \ldots\right)$. The Lax equation is

$$
\frac{\partial \mathcal{L}}{\partial t_{n}}=\left\{\left(\mathcal{L}^{n}\right)_{\geq 0}, \mathcal{L}\right\}
$$

Here the Poisson bracket is the same as in the dcmKP hierarchy. Also, the first equation $n=1$ of the hierarchy says that the dependence on $t_{1}$ and $x$ appears in the combination $t_{1}+x$. There exists a dressing function $\varphi=\sum_{n=1}^{\infty} \varphi_{n} k^{-n}$ such that

$$
\mathcal{L}=e^{\operatorname{ad} \varphi} k, \quad \quad \nabla_{t_{n}, \varphi} \varphi=-\left(\mathcal{L}^{n}\right)_{<0} .
$$

With this dressing function, the Orlov-Schulman function is defined by

$$
\mathcal{M}=e^{\operatorname{ad} \varphi}\left(\sum_{n=1}^{\infty} n t_{n} k^{n-1}+x\right)=\sum_{n=1}^{\infty} n t_{n} \mathcal{L}^{n-1}+x+\sum_{n=1}^{\infty} v_{n} \mathcal{L}^{-n-1} .
$$

There exists a tau function $\tau$ such that

$$
\frac{\partial \log \tau}{\partial t_{n}}=v_{n}
$$

We define the free energy by $\mathcal{F}=\log \tau$. 
3.3.2. Dispersionless Hirota equation. In terms of the tau function or the free energy, we have the following identities:

$$
\left(\mathcal{L}^{n}\right)_{\geq 0}=\mathcal{L}^{n}-\sum_{m=1}^{\infty} \frac{1}{m} \frac{\partial^{2} \mathcal{F}}{\partial t_{m} \partial t_{n}} \mathcal{L}^{-m}
$$

Now we identify $k$ with $w$ and $\mathcal{L}$ with $z$, the function $z=G(w)$ is defined to be $\mathcal{L}(k)$, and the function $w=g(z) \in \Sigma_{0}$ the inverse of $G(w)$. Then the Faber polynomials $\Phi_{n}(w)$ of $g$ are identified with $\left(\mathcal{L}^{n}(w)\right)_{\geq 0}$. Define the Grunsky coefficients of $g$ by equation (2.3), then comparing (2.4) with (3.23), we find the relation between the Grunsky coefficients and the free energy is given by

$$
b_{m n}=-\frac{1}{m n} \frac{\partial^{2} \mathcal{F}}{\partial t_{m} \partial t_{n}} .
$$

From the $n=1$ case of equation (3.23) and the fact that $(\mathcal{L})_{\geq 0}=k$, we have

$$
g(z)=z-\sum_{m=1}^{\infty} \frac{1}{m} \frac{\partial^{2} \mathcal{F}}{\partial t_{m} \partial t_{1}} z^{-m}
$$

Using this, and (3.24), we can rewrite the definition of the Grunsky coefficients (2.3) in terms of the free energy:

$$
1-\frac{1}{z_{1}-z_{2}} \sum_{m=1}^{\infty} \frac{z_{1}^{-m}-z_{2}^{-m}}{m} \frac{\partial^{2} \mathcal{F}}{\partial t_{m} \partial t_{1}}=\exp \left(\sum_{m, n=1}^{\infty} \frac{1}{m n} \frac{\partial^{2} \mathcal{F}}{\partial t_{m} \partial t_{n}} z_{1}^{-m} z_{2}^{-n}\right)
$$

which is the dispersionless Hirota equation for dKP.

Conversely, we can characterize the tau function for the dKP hierarchy as follows:

Proposition 3.5. If $\mathcal{F}=\log \tau$ is a function of $t_{n}, n \geq 1$ that satisfies the Hirota equation (3.25), then $\tau$ is a tau function of a solution of the $d K P$ hierarchy. More explicitly, if we define $\mathcal{L}(k)$ by formally inverting the function $\mathrm{k}(\mathcal{L})$ defined by

$$
\mathrm{k}(\mathcal{L})=\mathcal{L}-\sum_{m=1}^{\infty} \frac{1}{m} \frac{\partial^{2} \mathcal{F}}{\partial t_{1} \partial t_{m}} \mathcal{L}^{-m},
$$

and replacing $t_{1}$ by $t_{1}+x$, then $\mathcal{L}$ satisfies the Lax equations (3.22) for $d K P$.

Proof. The proof follows the same idea as Propositions 3.1 and 3.3 We define the function $g \in \Sigma_{0}$ by identifying $\mathcal{L}$ with $z$ in (3.26), namely

$$
g(z)=z-\sum_{m=1}^{\infty} \frac{1}{m} \frac{\partial^{2} \mathcal{F}}{\partial t_{1} \partial t_{m}} z^{-m}
$$


Hence the dispersionless Hirota equation (3.25) says that

$$
\log \frac{g\left(z_{1}\right)-g\left(z_{2}\right)}{z_{1}-z_{2}}=\sum_{m, n=1}^{\infty} \frac{1}{m n} \frac{\partial^{2} \mathcal{F}}{\partial t_{m} \partial t_{n}} z_{1}^{-m} z_{2}^{-n}
$$

Compare with the definition of the Grunsky coeffcients $b_{m n}$ (2.3) of $g$, we find that the $b_{m n}$ can be expressed in terms of the free energy by (3.24). Now define $z=G(w)$ to be the formal inverse of $w=g(z)$. In other words, $G(w)$ is $\mathcal{L}(k)$ if we identify $k$ with $w$. Then the Faber polynomials $\Phi_{n}(w)$ of $g$ are identified with $\left(\mathcal{L}^{n}(k)\right)_{\geq 0}$. Hence the identities satisfied by the Faber polynomials (2.4) can be rewritten as

$$
\left(\mathcal{L}^{n}\right)_{\geq 0}=\mathcal{L}^{n}-\sum_{m=1}^{\infty} \frac{1}{m} \frac{\partial^{2} \mathcal{F}}{\partial t_{m} \partial t_{n}} \mathcal{L}^{-m}
$$

Fixing $\mathcal{L}$, differentiate the $n=1$ case with respect to $t_{n}$ and compare to the result when we differentiate the $n$ case with respect to $t_{1}$, we have

$$
\left.\frac{\partial \mathrm{k}}{\partial t_{n}}\right|_{\mathcal{L} \text { fixed }}=-\sum_{m=1}^{\infty} \frac{1}{m} \frac{\partial^{3} \mathcal{F}}{\partial t_{m} \partial t_{1} \partial t_{n}} \mathcal{L}^{-m}=\left.\frac{\partial\left(\mathcal{L}^{n}\right)_{\geq 0}}{\partial t_{1}}\right|_{\mathcal{L} \text { fixed }}
$$

The same argument as in Proposition 3.3 gives the Lax equation (3.22) of dKP.

From this characterization of the tau functions, we can also see that a solution of the dcmKP hierarchy will give rise to a solution of the dKP hierarchy.

Corollary 3.6. If

$$
\mathcal{L}=k+\sum_{n=0}^{\infty} u_{n+1} k^{-n}
$$

is a solution of the dcmKP hierarchy, then

$$
\mathcal{L}^{\prime}=k+\sum_{n=1}^{\infty} u_{n+1}^{\prime} k^{-n}=k+\sum_{n=1}^{\infty} u_{n+1}\left(k-u_{1}\right)^{-n}
$$

is a solution of the dKP hierarchy, where we regard $t_{0}$ as a constant. Moreover, $\mathcal{L}$ and $\mathcal{L}^{\prime}$ have the same tau function.

Proof. In 6, 24, this was proved via a Miura map $\mathcal{L}^{\prime}=e^{\text {ad } \phi} \mathcal{L}$ to relate $\mathcal{L}$ and $\mathcal{L}^{\prime}$, where $\phi$ is defined in (3.13). Here we give a proof in terms of tau functions.

Since $\mathcal{L}=k+\sum_{n=0}^{\infty} u_{n+1} k^{-n}$ is a solution of the dcmKP, the free energy of $\mathcal{F}$ satisfies the dispersionless Hirota equation (3.18). Taking the limit $z_{2} \rightarrow \infty$ in (3.18), we obtain the relation

$$
z_{1} \exp \left(-\sum_{m=1}^{\infty} \frac{1}{m} \frac{\partial^{2} \mathcal{F}}{\partial t_{0} \partial t_{m}} z_{1}^{-m}\right)+\frac{\partial^{2} \mathcal{F}}{\partial t_{0} \partial t_{1}}=z_{1}-\sum_{m=1}^{\infty} \frac{1}{m} \frac{\partial^{2} \mathcal{F}}{\partial t_{m} \partial t_{1}} z_{1}^{-m},
$$


or

$$
z \exp \left(-\sum_{m=1}^{\infty} \frac{1}{m} \frac{\partial^{2} \mathcal{F}}{\partial t_{0} \partial t_{m}} z^{-m}\right)=z-\frac{\partial^{2} \mathcal{F}}{\partial t_{0} \partial t_{1}}-\sum_{m=1}^{\infty} \frac{1}{m} \frac{\partial^{2} \mathcal{F}}{\partial t_{m} \partial t_{1}} z^{-m} .
$$

Substituting this into the Hirota equation (3.18) again, we obtain

$$
z_{1}-z_{2}-\sum_{m=1}^{\infty} \frac{z_{1}^{m}-z_{2}^{m}}{m} \frac{\partial^{2} \mathcal{F}}{\partial t_{m} \partial t_{1}}=\left(z_{1}-z_{2}\right) \exp \left(\sum_{m, n=1}^{\infty} \frac{1}{m n} \frac{\partial^{2} \mathcal{F}}{\partial t_{m} \partial t_{n}} z_{1}^{-m} z_{2}^{-n}\right),
$$

which is equivalent to the dispersionless Hirota equation for dKP (3.25). Hence, from Proposition 3.5 above, the function $\mathcal{L}^{\prime}$ defined by inverting

$$
\mathrm{k}\left(\mathcal{L}^{\prime}\right)=\mathcal{L}^{\prime}-\sum_{m=1}^{\infty} \frac{1}{m} \frac{\partial^{2} \mathcal{F}}{\partial t_{m} \partial t_{1}}\left(\mathcal{L}^{\prime}\right)^{-m}
$$

is a solution of the dKP hierarchy. Now since the inverse $\mathrm{k}$ of $\mathcal{L}(k)$ satisfies

$$
\mathrm{k}(\mathcal{L})=\mathcal{L}-\frac{\partial^{2} \mathcal{F}}{\partial t_{0} \partial t_{1}}-\sum_{m=1}^{\infty} \frac{1}{m} \frac{\partial^{2} \mathcal{F}}{\partial t_{m} \partial t_{1}} \mathcal{L}^{-m}
$$

and $\frac{\partial^{2} \mathcal{F}}{\partial t_{0} \partial t_{1}}=u_{1}$, we find that

$$
\mathcal{L}^{\prime}=k+\sum_{n=1}^{\infty} u_{n+1}\left(k-u_{1}\right)^{-n}
$$

Remark 3.7. The proof of this corollary also shows that the dispersionless Hirota equation for dcmKP implies the dispersionless Hirota equation for dKP.

\section{Concluding Remarks}

We extend the definition of Grunsky coefficients and Faber polynomials formally to the space of formal power series. We have shown that the tau function or free energy of dToda, dcmKP and dKP hierarchies are closely related to Grunsky coefficients. We rederive the dispersionless Hirota equations and prove that they uniquely characterize the tau functions associated to a solution of the hierarchies. This might be helpful in classifying the solutions of the hierarchies. We also establish a link between these three dispersionless hierarchies. The transformation that relate the three versions of the dToda hierarchies and the Miura map that transform a solution of the dcmKP hierarchy to a solution of the dKP hierarchy are just the linear maps that relate the three spaces of formal power series we discuss in Section 2.

Given any formal power series $f \in \tilde{S}, g \in \Sigma$, if we define $\mathcal{F}_{m n}$ 's as

$$
\begin{aligned}
& \mathcal{F}_{m, n}=-|m n| b_{m, n}, \quad m \neq 0, n \neq 0, \\
& \mathcal{F}_{m, 0}=\mathcal{F}_{0, m}=|m| b_{m, 0}, \quad m \neq 0, \quad \mathcal{F}_{0,0}=-b_{0,0},
\end{aligned}
$$


where $b_{m, n}$ 's are the Grunsky coefficients associated to the pair $(f, g)$, then $\mathcal{F}_{m, n}$ 's satisfy the dispersionless Hirota equations (3.6), (3.18) and (3.25) if we replace $\frac{\partial^{2} \mathcal{F}}{\partial t_{m} \partial t_{n}}$ by $\mathcal{F}_{m, n}$. In [3], Sorin and Bonora proved that the Neumann coefficients that appear in string field theory satisfy the dispersionless Hirota bilinear equations. By definition, the Neumann coefficients coincide with the Grunsky coefficients $b_{m, n}$ defined above. This explain their results.

However, it is still an open question to find a function $\mathcal{F}$ such that

$$
\frac{\partial^{2} \mathcal{F}}{\partial t_{m} \partial t_{n}}=\mathcal{F}_{m, n}
$$

In [25], Wiegmann and Zabrodin provided a solution to this problem (see also [13, 16, 26]) when $G$, the inverse function of $g$ is an analytic function that maps the outer disc $\{|z|>1\}$ to the exterior of an analytic curve, and $f(z)=1 / \bar{g}\left(z^{-1}\right)$. It will be interesting to solve the general problem.

Acknowledgments. I am grateful to L. A. Takhtajan for his helpful comments. This work is partially supported by NSC grant NSC 91-2115-M-009017.

\section{REFERENCES}

1. L. Bonora and C. S. Xiong, Extended Toda lattice hierarchy, extended two-matrix model and $c=1$ string theory, Nuclear Phys. B 434 (1995), no. 1-2, 408-444.

2. — Two-matrix model and $c=1$ string theory, Phys. Lett. B 347 (1995), no. 1-2, $41-48$.

3. Sorin A.S. Bonora, L., Integrable structures in string field theory, Preprint arXiv: hep-th/0211283 (2002).

4. A. Boyarsky, A. Marshakov, O. Ruchayskiy, P. Wiegmann, and A. Zabrodin, Associativity equations in dispersionless integrable hierarchies, Phys. Lett. B 515 (2001), no. 3-4, 483-492.

5. R. Carroll and Y. Kodama, Solution of the dispersionless Hirota equations, J. Phys. A 28 (1995), no. 22, 6373-6387.

6. J. H. Chang and M. H. Tu, On the Miura map between the dispersionless KP and dispersionless modified KP hierarchies, J. Math. Phys. 41 (2000), no. 8, 5391-5406.

7. L. de Branges, A proof of the Bieberbach conjecture, Acta Math. 154 (1985), no. 1-2, 137-152.

8. B. Dubrovin, Integrable systems in topological field theory, Nuclear Phys. B 379 (1992), no. 3, 627-689.

9. Integrable systems and classification of 2-dimensional topological field theories, Integrable systems (Luminy, 1991), Progr. Math., vol. 115, Birkhäuser Boston, Boston, MA, 1993, pp. 313-359.

10. _ Geometry of $2 D$ topological field theories, Integrable systems and quantum groups (Montecatini Terme, 1993), Lecture Notes in Math., vol. 1620, Springer, Berlin, 1996, pp. $120-348$.

11. B. A. Dubrovin, Hamiltonian formalism of Whitham-type hierarchies and topological Landau-Ginsburg models, Comm. Math. Phys. 145 (1992), no. 1, 195-207.

12. P. L. Duren, Univalent functions, Grundlehren der Mathematischen Wissenschaften [Fundamental Principles of Mathematical Sciences], vol. 259, Springer-Verlag, New York, 1983.

13. I. K. Kostov, I. M. Krichever, M. Mineev-Weinstein, P. B. Wiegmann, and A. Zabrodin, The $\tau$-function for analytic curves, Random matrix models and their 
applications, Math. Sci. Res. Inst. Publ., vol. 40, Cambridge Univ. Press, Cambridge, 2001, pp. 285-299.

14. I. M. Krichever, The dispersionless Lax equations and topological minimal models, Comm. Math. Phys. 143 (1992), no. 2, 415-429.

15. - The $\tau$-function of the universal Whitham hierarchy, matrix models and topological field theories, Comm. Pure Appl. Math. 47 (1994), no. 4, 437-475.

16. A. Marshakov, P. Wiegmann, and A. Zabrodin, Integrable structure of the Dirichlet boundary problem in two dimensions, Comm. Math. Phys. 227 (2002), no. 1, 131-153.

17. C. Pommerenke, Univalent functions, Vandenhoeck \& Ruprecht, Göttingen, 1975, With a chapter on quadratic differentials by Gerd Jensen, Studia Mathematica/Mathematische Lehrbücher, Band XXV.

18. K. Takasaki, Dispersionless Toda hierarchy and two-dimensional string theory, Comm. Math. Phys. 170 (1995), no. 1, 101-116.

19. Integrable hierarchies, dispersionless limit and string equations, Structure of solutions of differential equations (Katata/Kyoto, 1995), World Sci. Publishing, River Edge, NJ, 1996, pp. 457-481.

20. K. Takasaki and T. Takebe, SDiff(2) Toda equation-hierarchy, tau function, and symmetries, Lett. Math. Phys. 23 (1991), no. 3, 205-214.

21. Math. Phys., vol. 16, World Sci. Publishing, River Edge, NJ, 1992, pp. 889-922.

22. Luasi-classical limit of Toda hierarchy and W-infinity symmetries, Lett. Math. Phys. 28 (1993), no. 3, 165-176.

23. _ Integrable hierarchies and dispersionless limit, Rev. Math. Phys. 7 (1995), no. $5,743-808$.

24. L. P. Teo, On dispersionless coupled modified KP hierarchy, Preprint arXiv: nlin.SI/0304007.

25. P. B. Wiegmann and A. Zabrodin, Conformal maps and integrable hierarchies, Comm. Math. Phys. 213 (2000), no. 3, 523-538.

26. A. V. Zabrodin, The dispersionless limit of the Hirota equations in some problems of complex analysis, Teoret. Mat. Fiz. 129 (2001), no. 2, 239-257.

Department of Applied Mathematics, National Chiao Tung University, 1001, Ta-Hsueh Road, Hsinchu City, 30050, Taiwan, R.O.C.

E-mail address: lpteo@math.nctu.edu.tw 